\title{
Should sarcopenia be an additional factor enough to affect liver transplant decision-making?
}

\author{
Kang He, Qiang Xia \\ Department of Liver Surgery, Renji Hospital, School of Medicine, Shanghai Jiao Tong University, Shanghai, China \\ Correspondence to: Qiang Xia. Department of Liver Surgery, Renji Hospital, School of Medicine, Shanghai Jiao Tong University, Shanghai, China. \\ Email: xiaqiang@shsmu.edu.cn. \\ Comment on: Pravisani R, Soyama A, Ono S, et al. Is there any correlation between liver graft regeneration and recipient's pretransplant skeletal \\ muscle mass?-a study in extended left lobe graft living-donor liver transplantation. Hepatobiliary Surg Nutr 2020;9:183-94.
}

Submitted Aug 23, 2021. Accepted for publication Sep 23, 2021.

doi: 10.21037/hbsn-2021-19

View this article at: https://dx.doi.org/10.21037/hbsn-2021-19

Sarcopenia is defined as the loss of muscle mass and function and is now recognized in all types of chronic diseases, including end-stage liver diseases. Sarcopenia increases the risk of morbidity and mortality before and after liver transplantation (LT) (1). The risks associated with the condition are greater in living donor LT (LDLT) recipients, as low model for end-stage liver disease (MELD) scores associated with LDLT can be masked by sarcopenia, leading to increased mortality and sepsis after LT (2).

Seeking to determine a connection between liver graft regeneration and pre-LDLT sarcopenia in patients, Pravisani $e t$ al. conducted a retrospective study on analysis of their relationship in LDLT recipients. The results showed that pre-LDLT sarcopenia was associated with a decreased graft regeneration rate (GRR) and that this negative effect was more pronounced among male patients (3).

The study showed that the median GRR at 1 month post-LDLT was $91 \%$, and a significant correlation between GRR and pre-LDLT low muscularity was noted. A low GRR was regarded as an independent predictor for the overall survival of recipients. The study also demonstrated that sarcopenia can influence the prognosis of LT patients. Although it showed the importance of sarcopenia in LT recipient outcomes, the study also raised the question of whether sarcopenia should be a factor in the decisionmaking process for liver transplants.

Despite the poor outcomes related to the presence of sarcopenia, current practice does not take into account a patient's physical condition when prioritizing patients for LT. Sarcopenia exists in almost $50 \%$ of cirrhotic patients and increases a patient's vulnerability to complications. The appropriate quantification of sarcopenia can improve preLT evaluation and allow for early intervention to improve patient outcomes after LT (4).

To address this question, the core principles of cirrhotic patients' prioritization for LT should be outlined. Generally, MELD scores and, more recently, MELD Sodium (MELDNa) scores have been used as criteria for decision-making when prioritizing LT candidates. These scores have proven most useful in selecting the "sickestfirst" candidates for LT based on the predicted 3-month mortality rate. However, more than $75 \%$ of patients with liver disease present with protein-calorie malnutrition, the severity of which rises with the severity of the complications associated with the disease, including sarcopenia. Despite this, sarcopenia is frequently overlooked in these cases, leading to lost opportunities for LT and worse post-LT outcomes, independent of the MELD scores (5).

One study suggested adding a sarcopenia index to the existing MELD scoring system to improve risk predictions based on MELD or MELDNa scores alone by indirectly explaining the clinical manifestations of sarcopenia. However, this MELD-Sarcopenia score only demonstrated a better prediction rate of mortality in patients with cirrhosis, and primarily in those with low MELD scores $(<15)$ (6). Another study suggested that in sarcopenic patients with MELDNa <20, an "urgency" Sarco-Model 2 , which combines scores for sarcopenia and MELDNa, could be used to prioritize waiting lists for LT, while a MELDNa value alone might be preferable for patients with MELDNa 
$\geq 20$. The study also proposed that for sarcopenic patients with a MELDNa value of 35-40, "futile" transplantation should be considered (7). According to the American Association for the Study of Liver Diseases (AASLD) 2021 Practice Guidance on the management of malnutrition, frailty, and sarcopenia in patients with cirrhosis, objective, standardized metrics for sarcopenia should be taken into account for LT candidates with the condition (8).

It is worth noting that to date, most studies have used static measures of sarcopenia. However, recent reports demonstrate that sarcopenia is progressive and that dynamic assessment of muscle loss rates from continuous or longitudinal measures can help predict patient outcomes. It was recommended that sarcopenia be reassessed every 8 to 12 weeks in patients with decompensated cirrhosis and in patients undergoing aggressive treatment for sarcopenia $(8,9)$. Another interesting study demonstrated that in patients with end-stage liver disease who were waiting for LT, lower muscle quality, or muscle attenuation, and sarcopenia correlated independently with an ascending risk of death, suggesting that besides sarcopenia, muscle attenuation may have an additional value as a risk predictor in patients who are at risk of death while waiting for LTs (10).

Although patients displayed rates of muscle mass improvement from $25 \%$ to $34 \%$ after LT, it found that $26 \%$ of LT recipients demonstrated new-onset sarcopenia after LT. These results suggest that while LT may help in improving a recipient's sarcopenia, it cannot be recommended as a specific treatment for sarcopenia. In addition, liver dysfunction is not the only cause of muscle loss. Long-term cirrhosis may permanently change skeletal muscle, resulting in persistent sarcopenia that cannot simply be reversed by LT (11). Despite the lack of data indicating the specific thresholds for objective indicators of sarcopenia that compromises risk of waitlist with post-LT death, taking sarcopenia as an absolute contraindication to LT is not recommended.

A growing number of studies highlight the importance of sarcopenia as a prognostic marker for patients with cirrhosis, paving the way for future research. Multimodal strategies targeting sarcopenia and its impact on decisionmaking for LT candidates are needed. Thus, it would be ideal for trained and multidisciplinary caregivers to incorporate the evaluation and management of hepatology, surgery, nutrition, and physiotherapy for candidates on waiting lists for LT. Sarcopenia alone is unlikely to drive decision-making regarding transplants for patients with multiple favorable or mitigating characteristics, and adding sarcopenia into a scoring system for prioritizing waiting lists will require cooperation from all sectors, including academia, organ allocation systems, and policymakers. More prospective studies must be undertaken and methodological concerns addressed Before scoring systems that incorporate sarcopenia can be applied in clinical practice.

\section{Acknowledgments}

Funding: None.

\section{Footnote}

Provenance and Peer Review: This article was commissioned by the editorial office, Hepatobiliary Surgery and Nutrition. The article did not undergo external peer review.

Conflicts of Interest: Both authors have completed the ICMJE uniform disclosure form (available at https://hbsn. amegroups.com/article/view/10.21037/hbsn-2021-19/coif). Dr. QX serves as the unpaid editorial board member of Hepatobiliary Surgery and Nutrition. The other author has no conflicts of interest to declare.

Etbical Statement: The authors are accountable for all aspects of the work in ensuring that questions related to the accuracy or integrity of any part of the work are appropriately investigated and resolved.

Open Access Statement: This is an Open Access article distributed in accordance with the Creative Commons Attribution-NonCommercial-NoDerivs 4.0 International License (CC BY-NC-ND 4.0), which permits the noncommercial replication and distribution of the article with the strict proviso that no changes or edits are made and the original work is properly cited (including links to both the formal publication through the relevant DOI and the license). See: https://creativecommons.org/licenses/by-nc-nd/4.0/.

\section{References}

1. Saiman Y, Serper M. Frailty and Sarcopenia in Patients Preand Post-Liver Transplant. Clin Liver Dis 2021;25:35-51.

2. Masuda T, Shirabe K, Ikegami T, et al. Sarcopenia is a prognostic factor in living donor liver transplantation. Liver Transpl 2014;20:401-7.

3. Pravisani R, Soyama A, Ono S, et al. Is there any correlation between liver graft regeneration and recipient's 
pretransplant skeletal muscle mass?-a study in extended left lobe graft living-donor liver transplantation. Hepatobiliary Surg Nutr 2020;9:183-94.

4. Haugen CE, McAdams-DeMarco M, Holscher CM, et al. Multicenter Study of Age, Frailty, and Waitlist Mortality Among Liver Transplant Candidates. Ann Surg 2020;271:1132-6.

5. Wu Y, Zhu Y, Feng Y, et al. Royal Free HospitalNutritional Prioritizing Tool improves the prediction of malnutrition risk outcomes in liver cirrhosis patients compared with Nutritional Risk Screening 2002. Br J Nutr 2020;124:1293-302.

6. Montano-Loza AJ, Duarte-Rojo A, Meza-Junco J, et al. Inclusion of Sarcopenia Within MELD (MELDSarcopenia) and the Prediction of Mortality in Patients With Cirrhosis. Clin Transl Gastroenterol 2015;6:e102.

7. Lai Q, Magistri P, Lionetti R, et al. Sarco-Model: A score

Cite this article as: $\mathrm{He} \mathrm{K}, \mathrm{Xia} \mathrm{Q}$. Should sarcopenia be an additional factor enough to affect liver transplant decisionmaking? HepatoBiliary Surg Nutr 2021;10(6):884-886. doi: 10.21037/hbsn-2021-19 to predict the dropout risk in the perspective of organ allocation in patients awaiting liver transplantation. Liver Int 2021;41:1629-40.

8. Lai JC, Tandon P, Bernal W, et al. Malnutrition, Frailty, and Sarcopenia in Patients With Cirrhosis: 2021 Practice Guidance by the American Association for the Study of Liver Diseases. Hepatology 2021;74:1611-44.

9. Welch N, Dasarathy J, Runkana A, et al. Continued muscle loss increases mortality in cirrhosis: Impact of aetiology of liver disease. Liver Int 2020;40:1178-88.

10. Bot D, Droop A, Lucassen CJ, et al. Both muscle quantity and quality are predictors of waiting list mortality in patients with end-stage liver disease. Clin Nutr ESPEN 2021;42:272-9.

11. Tsien C, Garber A, Narayanan A, et al. Post-liver transplantation sarcopenia in cirrhosis: a prospective evaluation. J Gastroenterol Hepatol 2014;29:1250-7. 